\title{
Efficacy and safety of concurrent anti-tuberculosis treatment and chemotherapy in lung cancer patients with co-existent tuberculosis
}

\author{
Mei-Feng Ye ${ }^{1 \#}$, Shan Su ${ }^{2 \#}$, Zhi-Hao Huang ${ }^{2 \#}$, Jian-Jun Zou ${ }^{2}$, Duo-Hua Su${ }^{3}$, Xiao-Hui Chen ${ }^{4}$, \\ Long-Feng Zeng ${ }^{3}$, Wei-Xiang Liao ${ }^{3}$, Hui-Yi Huang ${ }^{2}$, Yun-Yun Zeng ${ }^{2}$, Wen-Chang Cen ${ }^{2}$, \\ Xian-Lan Zhang ${ }^{2}$, Chun-Xin Liao ${ }^{3}$, Jian Zhang ${ }^{5}$, Yan-Bin Zhang ${ }^{3}$ \\ ${ }^{1}$ Guangzhou Medical University, Guangzhou, China; ${ }^{2}$ Department of Oncology, Guangzhou Chest Hospital, Guangzhou, China; ${ }^{3}$ Department of \\ Tuberculosis, Guangzhou Chest Hospital, Guangzhou, China; ${ }^{4}$ Department of Thoracic Surgery, Guangzhou Chest Hospital, Guangzhou, China; \\ ${ }^{5}$ Department of Oncology, Zhujiang Hospital, Guangzhou, China \\ Contributions: (I) Conception and design: S Su, YB Zhang; (II) Administrative support: YB Zhang, J Zhang; (III) Provision of study materials or \\ patients: JJ Zou, DH Su, XH Chen, LF Zeng, WX Liao, HY Huang, YY Zeng, WC Cen, XL Zhang, CX Liao; (IV) Collection and assembly of data: \\ ZH Huang, MF Ye; (V) Data analysis and interpretation: S Su, MF Ye; (VI) Manuscript writing: All authors; (VII) Final approval of manuscript: All \\ authors. \\ \#These authors contributed equally to this work. \\ Correspondence to: Prof. Yan-Bin Zhang. Guangzhou Chest Hospital, 62 Hengzhigang Rd., Guangzhou, China. Email: drzhangyb@hotmail.com; Prof. \\ Jian Zhang. Zhujiang Hospital, 253 Gongye Rd., Guangzhou, China. Email: blacktiger@139.com.
}

Background: This retrospective study evaluated the safety and efficacy of concurrent anti-tuberculosis (TB) and chemotherapy treatment in patients with advanced lung cancer and active TB.

Methods: We retrospectively analyzed patients who were first diagnosed with advanced lung cancer and received first-line chemotherapy in Guangzhou Chest Hospital from 2015 to 2017. Patients were categorized into two groups (2:1): lung cancer patients without active TB (Group A), and lung cancer patients with active TB (Group B). Primary endpoints included adverse events (AEs), objective response rate (ORR), time to treatment failure, and overall survival (OS).

Results: A total of 99 patients were eligible (Group A, n=66; Group B, n=33). Grade $\geq 3$ treatmentrelated AEs, primarily hematologic toxicity, occurred in $39.4 \%$ and $51.5 \%$ of patients in Groups A and B, respectively. The hypohepatia in both groups was generally at grade 1 or 2 , with similar incidences $(26 \%$ and $27 \%$, respectively). After two cycles of chemotherapy, the ORR was $42.4 \%$ and $33.3 \%$ in Group A and B, respectively $(\mathrm{P}=0.383)$. The median time to treatment failure (TTF) was 7.0 and 5.6 months for Groups A and $\mathrm{B}$, respectively $(\mathrm{P}=0.175)$. The median $\mathrm{OS}$ was 17.0 and 14.0 months for Groups $\mathrm{A}$ and $\mathrm{B}$, respectively $(\mathrm{P}=0.312)$. After 3 months of anti-TB treatment, all patients achieved sputum acid-fast bacilli (AFB) smear conversion and absorption on imaging, and the end of follow-up observed no recurrence.

Conclusions: Concurrent anti-TB and chemotherapy treatment did not increase hematological toxicity or hypohepatia in lung cancer patients with pulmonary TB.

Keywords: Lung cancer; pulmonary tuberculosis (pulmonary TB); chemotherapy

Submitted Jun 23, 2020. Accepted for publication Sep 04, 2020.

doi: 10.21037/atm-20-5964

View this article at: http://dx.doi.org/10.21037/atm-20-5964 


\section{Introduction}

Lung cancer with tuberculosis (TB) was first reported in the 19C. Since then, extensive research has been conducted on the co-existence of these two diseases. Immunodeficiency due to lung cancer treatment can cause the reactivation of $\mathrm{TB}$, while TB increases lung cancer risk due to confounding factors (1-3). Data from Japan from the last twenty years estimates that $1-2 \%$ of lung cancer patients have active TB $(4,5)$. The incidence may be higher in countries with a higher TB burden.

Anti-TB drugs, such as isoniazid and rifampicin, can cause a decline in white blood cells and platelets, and damage liver function, which are also common adverse effects of chemotherapy (6-8). Most previous studies have been limited to the epidemiology of co-existent TB and lung cancer and the relationship between them. Few studies have examined the efficacy and safety of concurrent chemotherapy and anti-TB treatment. A South Korean study found that anti-cancer chemotherapy is not an obstacle to treating TB (9). A study from Japan focused on patients with malignancy, and active TB suggested that concurrent chemotherapy and anti-TB treatment is effective and safe (10). However, due to the small sample size, the diversity of tumor types, and lack of controls, there are few evidence-based recommendations for combination treatment. China, one of the top 30 countries globally in terms of TB burden, has a consistently high incidence of lung cancer (11). Thus, it is of particular importance to China to determine the optimal strategy for treating lung cancer patients with co-existent $\mathrm{TB}$, and beneficial for other areas where both $\mathrm{TB}$ and lung cancer are prevalent.

The present study explored the efficacy and safety of concurrent chemotherapy and anti-TB treatment in lung cancer patients with active TB in China. The clinical outcomes of these patients were also explored.

We present the following article in accordance with the STROBE reporting checklist (available at http://dx.doi. org/10.21037/atm-20-5964).

\section{Methods}

\section{Study population}

We retrospectively analyzed patients who were first diagnosed with advanced lung cancer and received first-line chemotherapy in Guangzhou Chest Hospital from 2015 to 2017. For patients records retrieved from hospital electronic medical record system, data included age, gender, tuberculosis diagnosis, tumor stage, pathological type, adverse events, treatment information (include anti-TB treatment and chemotherapy), and survival data. Patients with stage I-III cancer, positive driver gene mutations (EGFR, ALK, or ROS1), multiple chemotherapies, or absence of treatment information was excluded. Ninetynine eligible patients were identified and propensity score matched into two groups (2:1): patients without co-existence of active pulmonary TB (Group A; $n=66$ ) and patients with co-existence of active pulmonary TB (Group B; $n=33$ ) (Figure 1). Variables used for propensity matching were as follows: age, sex, smoking history, ECOG performance status, disease stage and pathology. Patient characteristics at the baseline are summarized in Table 1.

All procedures performed in this study involving human participants were in accordance with the Declaration of Helsinki (as revised in 2013). The study was approved by the ethics committee of Guangzhou Chest Hospital (No. 2020-21). The requirement of informed consent was waived because of the retrospective nature of the study.

\section{Diagnosis of $T B$}

Active pulmonary TB was diagnosed in several ways:

Positive smear test for acid-fast bacilli (AFB) in sputum specimen or bronchial washing. In smear-negative specimens, a follow-on test of mycobacteria using a WHOapproved rapid diagnostic reagent, e.g., Xpert MTB/RIF, was performed.

Pathological diagnosis of granulomatous inflammation obtained by bronchoscopy or percutaneous lung biopsy (PTLB).

For patients who could not be diagnosed by microbiology or pathology, pulmonary TB was diagnosed based on typical pulmonary TB clinical symptoms, manifestations on chest X-ray, and effectiveness of anti-TB treatment after excluding other pulmonary diseases.

\section{Procedures}

Chemotherapy regimens for different pathological types of lung cancer are shown in Table 2. Regimens and pathology types were well-balanced between the two groups. Most patients received 21-day cycles of first-line platinum-based chemotherapy, including pemetrexed, paclitaxel, etoposide, and gemcitabine. One patient in each group received a 28 -day cycle of oral tegafur monotherapy due to poor physical condition (ECOG performance status $=3$ ). 


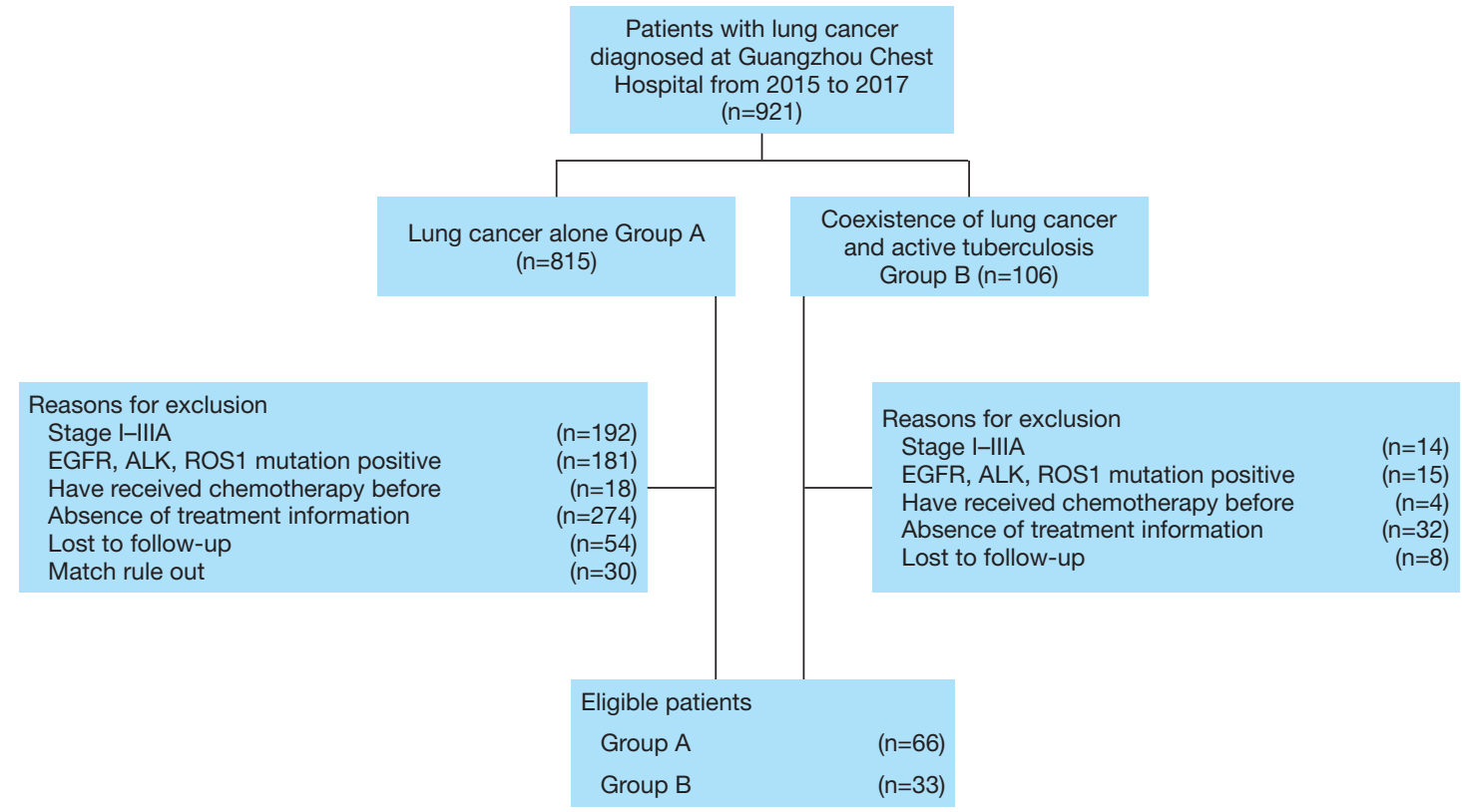

Figure 1 Flow chart of patient eligibility.

Patients with active TB (Group B) initially received a rifampicin-based anti-TB treatment regimen, including isoniazid $(\mathrm{H})$, rifampicin $(\mathrm{R})$, pyrazinamide $(\mathrm{Z})$, and ethambutol (E). All patients received 2-3 months of intensive treatment and 4-6 months of consolidation treatment.

\section{Evaluation of efficacy and safety}

Chest computed tomography (CT) was carried out every two chemotherapy cycles to determine tumor response and disease progression. Short-term efficacy was evaluated based on tumor response, classified as complete response (CR), partial response (PR), stable disease (SD), or progressive disease (PD) according to the Response Evaluation Criteria in Solid Tumors (RECIST 1.1) (12). Objective response rate (ORR) was the percentage of patients with CR and PR. Disease control rate (DCR) was the percentage of patients with CR, PR, and SD. Time to treatment failure (TTF) was defined as the time from initiation of chemotherapy until discontinuation of first-line chemotherapy due to PD, therapeutic toxicity, patient preference, or death. Overall survival (OS) was defined as the interval from pathological diagnosis to death for any reason. Patients who survived or who were lost to follow-up were censored at the time of the last follow-up.
Safety was measured by comparing the incidence of treatment-related adverse events (AEs) in each group. AEs were graded according to the National Cancer Institute Common Terminology Criteria for Adverse Events, Version 5.0.

\section{Statistical analysis}

Differences in demographics, clinicopathological characteristics, AEs, and ORR were assessed using the Chisquared or Fisher's exact tests. TTF and OS were evaluated by the Kaplan-Meier method and compared with results generated using the log-rank test. Statistical analyses were performed using SPSS version 22.0. All reported P values were two-tailed, and differences were considered statistically significant at $\mathrm{P}<0.05$.

\section{Results}

\section{Efficacy of anti-TB treatment}

Of the 33 patients in Group B, ten started chemotherapy within 2 weeks of anti-TB treatment, and 23 received chemotherapy after 2 weeks of anti-TB treatment. After 3 months of anti-TB treatment, all patients achieved sputum AFB smear conversion and absorption on imaging. The end of follow-up observed no recurrence. 
Table 1 Demographics and baseline patient characteristics

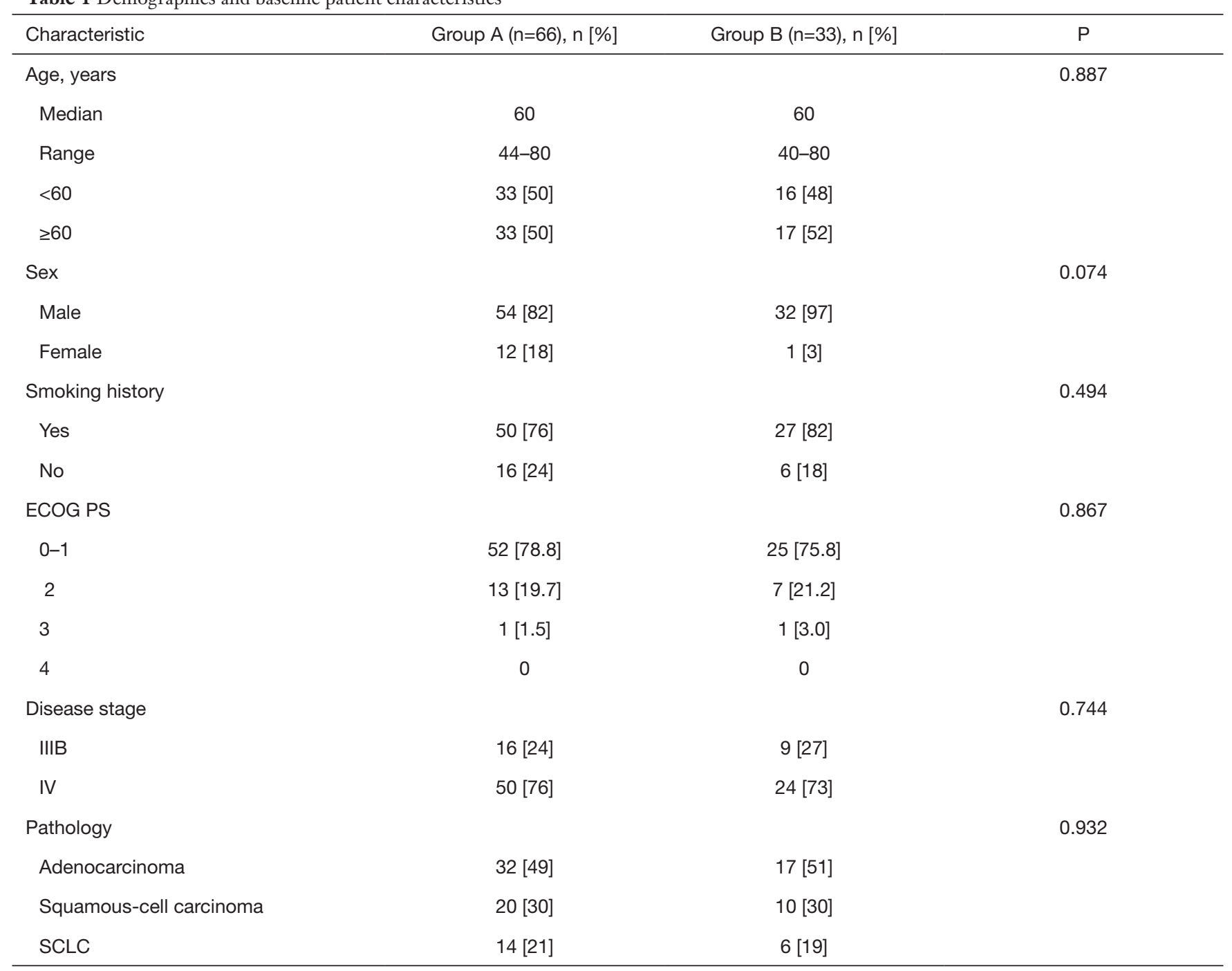

SCLC, small cell lung cancer; ECOG PS, Eastern Cooperative Oncology Group performance status.

\section{Efficacy of chemotherapy}

Three patients were excluded from analysis due to a lack of assessment data. The efficacy of chemotherapy was evaluated in the remaining 96 patients (Table 3). ORRs were 42.4\% (28 patients) and 33.3\% (11 patients) in Group A and Group B, respectively $(\mathrm{P}=0.383)$. DCRs in either group were not statistically different $(81.8 \%$ vs. $72.7 \%$ in Group A and $\mathrm{B}$ respectively; $\mathrm{P}=0.297$ ), irrespective of pathology type, age, sex, smoking status, or ECOG performance status.

At the cutoff date of March 1, 2019, seven patients $(10.6 \%)$ in Group A remained on anti-tumor treatment, three patients $(4.5 \%)$ had discontinued treatment due to AEs, and $52(78.8 \%)$ patients had discontinued treatment due to disease progression.

In Group B, 27 patients (82\%) had discontinued therapy due to disease progression, and one patient had discontinued treatment due to grade 3 neutropenia and grade 4 thrombocytopenia.

Patients in Group A had a similar treatment time to Group B (median TTF 7.0 vs. 5.6 months, respectively; HR, 0.74; 95\% CI, 0.45-1.19; $\mathrm{P}=0.175$ ) (Figure 2A). We conducted an exploratory subgroup analysis of the TTF review according to pre-specified baseline characteristics; the results were generally consistent with the overall population (Figure 2B).

At the cutoff date of March 1, 2019, 46 patients (69.7\%) 
Table 2 Chemotherapy regimens for different pathological types of lung cancer

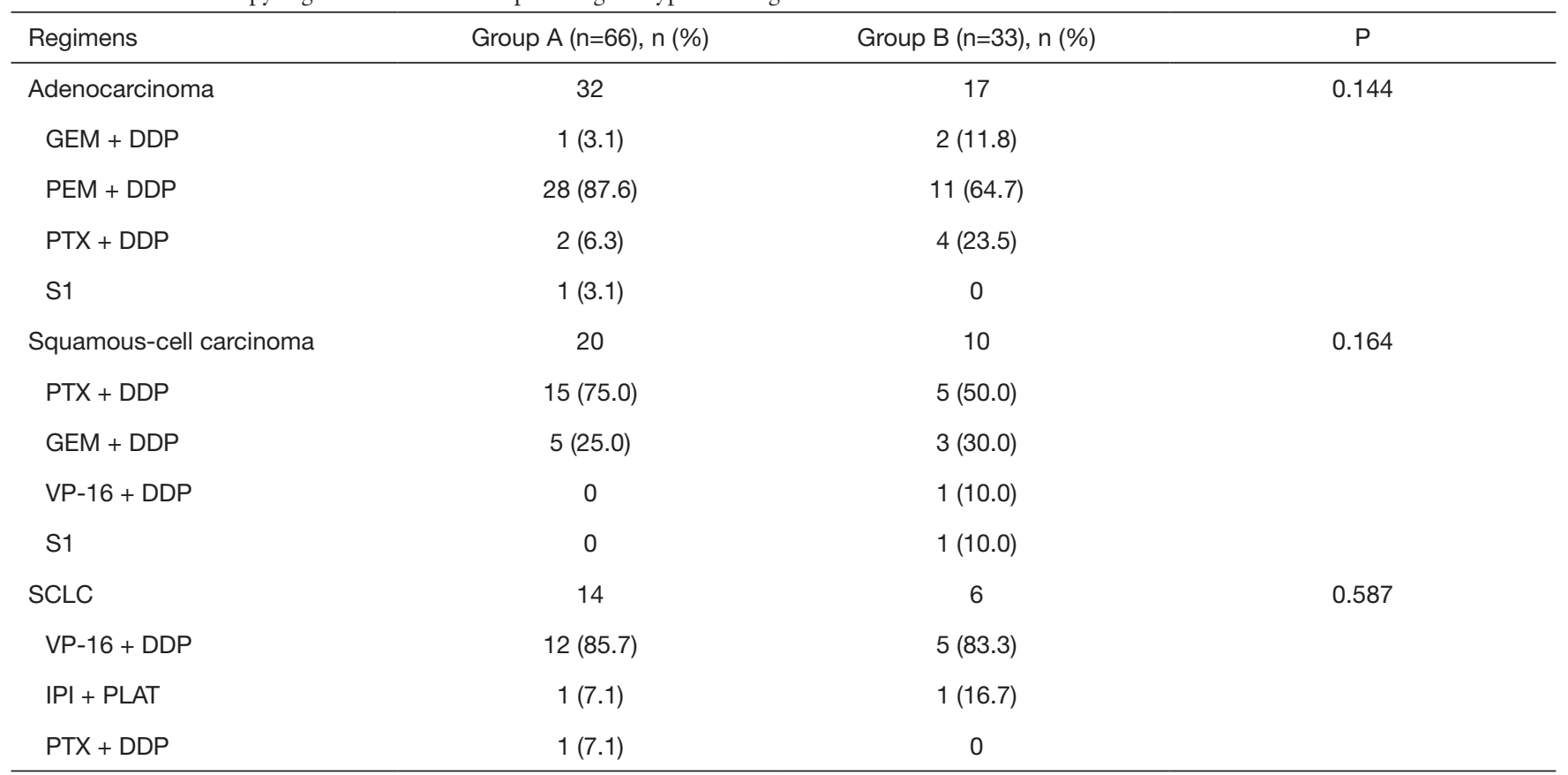

GEM, gemcitabine; PEM, pemetrexed; PTX, paclitaxel; DDP, Cisplatin; S1, tegafur; SCLC, small cell lung cancer; VP-16, Etoposide; IPI, irinotecan.

Table 3 Tumor response rates after two chemotherapy cycles

\begin{tabular}{|c|c|c|c|}
\hline Tumor response rates & Group A (n=66), n (\%) & Group B (n=33), n (\%) & $\mathrm{P}$ \\
\hline PR & $27(40.9)$ & $11(33.3)$ & \\
\hline SD & $26(39.4)$ & $13(39.4)$ & \\
\hline PD & $10(15.2)$ & $8(24.3)$ & \\
\hline ORR (CR + PR) & $28(42.4)$ & $11(33.3)$ & 0.383 \\
\hline $\mathrm{DCR}(\mathrm{CR}+\mathrm{PR}+\mathrm{SD})$ & $54(81.8)$ & $24(72.7)$ & 0.297 \\
\hline
\end{tabular}

CR, complete response; PR, partial response; SD, stable disease; PD, progressive disease; ORR, objective response rate; DCR, disease control rate.

in Group A and 28 (84.8\%) in Group B had died. The median duration of follow-up for OS was 8 and 6 months in Group A and B, respectively. Median OS in Group A was 17 months (95\% CI, 9.7-24.3 months) and in Group B was 14 months (95\% CI, $10.5-17.5$ months) with no statistically significant difference in OS (HR, 0.76; 95\% CI, 0.44-1.33; $\mathrm{P}=0.312$ ) (Figure 2C). In the univariate analysis, age, performance status, smoking status, and pathology were not significantly associated with OS (Figure 2D).

\section{Safety of concurrent anti-TB treatment and chemotherapy}

The incidence of AEs of any cause was $84.8 \%(56 / 66)$ in Group A and 97\% (32/33) in Group B (Table 4). In Group A, the most frequently reported AEs of any grade were anemia (69.7\%), neutropenia (53.0\%), thrombocytopenia (24.2\%), and hypohepatia $(25.8 \%)$ and in Group B, anemia (81.8\%), neutropenia $(54.6 \%)$, thrombocytopenia $(18.2 \%)$, and hypohepatia $(27.3 \%)$. The frequency of grade $\geq 3$ treatmentrelated AEs of any cause was similar in both groups (39.4\% 

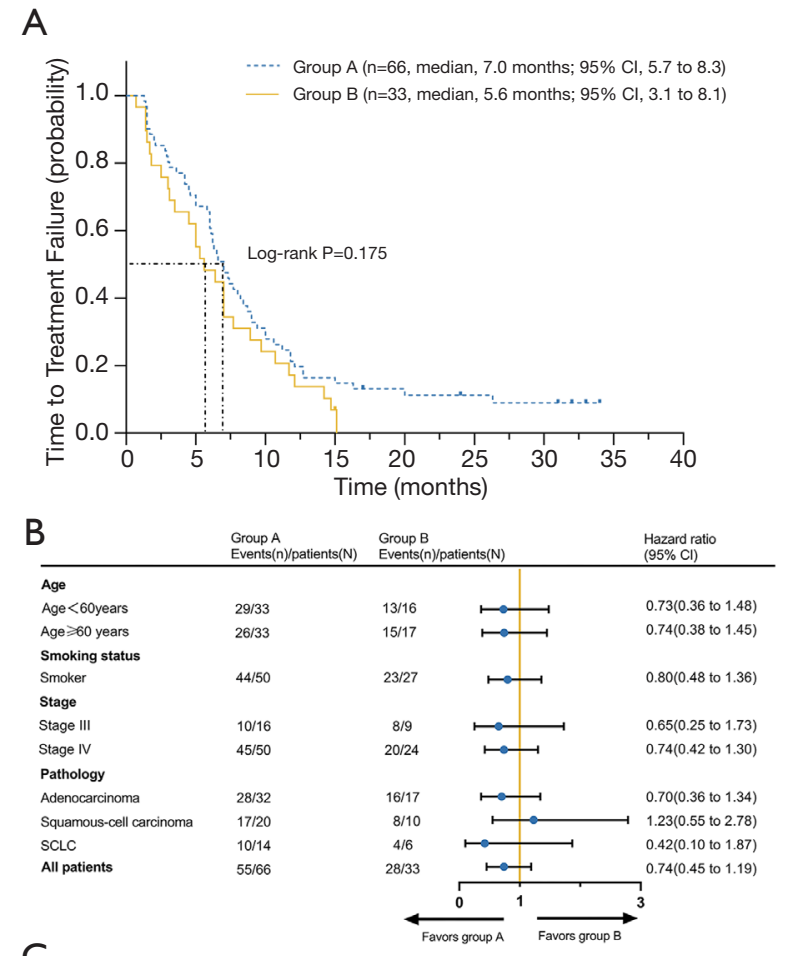

C
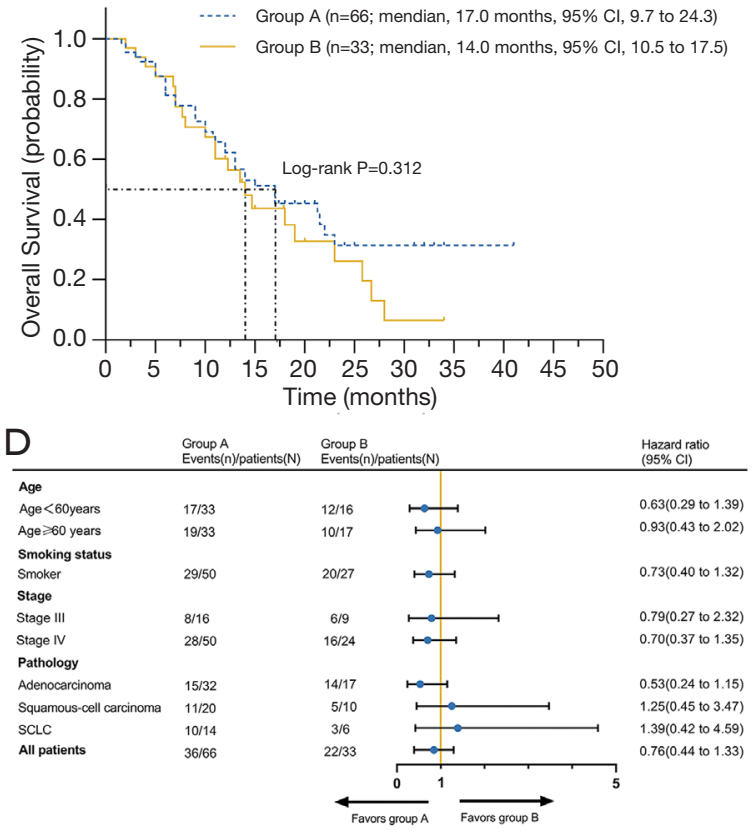

Figure 2 (A) Kaplan-Meier curve of TTF. (B) Forest plot depicting exploratory subgroup analyses effect on TTF. (C) Kaplan-Meier curve of OS. (D) Forest plot depicting exploratory subgroup analyses effect on OS (Group A vs. Group B). OS, overall survival; TTF, time to treatment failure. and $51.5 \%$ in Group A and B, respectively). Among these, the most common grade $\geq 3$ treatment-related AEs were hematologic toxicity, including comprised neutropenia (25.8\% vs. $24.2 \%$ in Groups $\mathrm{A}$ and $\mathrm{B}$, respectively; $\mathrm{P}=0.87)$ and anemia $(24.2 \%$ vs. $33.3 \%$ in Groups $\mathrm{A}$ and $\mathrm{B}$, respectively; $\mathrm{P}=0.338)$. Only one case with grade $\geq 3$ hypohepatia occurred in Group B. In subgroup analysis, a higher incidence of anemia was observed in patients over 60 years of age in Group B (see Table S1). There was no significant difference in the incidence of AEs between the subgroups based on the baseline characteristics.

Four patients in each group received a reduced chemotherapy dose due to grade $\geq 3$ neutropenia. One patient in Group A and three patients in Group B received blood transfusions for grade 3 anemia. One patient in Group B discontinued chemotherapy and changed the antiTB regimen due to grade 3 hypohepatia. No treatmentrelated $\mathrm{AE}$ deaths occurred in either group.

\section{Discussion}

Three main theories exist for the co-existence of lung cancer and TB: anti-cancer therapy can cause reactivation of TB; TB is a predisposing factor for lung cancer; or, it is random $(13,14)$. However, there is no standard treatment regimen for lung cancer patients with TB. The present study aimed to demonstrate the efficacy and safety of concurrent chemotherapy and anti-TB treatment. Our retrospective analysis revealed that anti-TB treatment in patients with co-existent TB and lung cancer did not affect chemotherapy efficacy and effectively controlled TB. Furthermore, the incidence of AEs in these patients did not increase. To the best of our knowledge, this is the first study with a large sample size to compare the efficacy and safety of concurrent anti-TB treatment and chemotherapy versus chemotherapy alone.

Several previous studies have focused on the efficacy and AEs of concurrent chemotherapy and anti-TB treatment in cancer patients with active TB. Kim et al. observed that chemotherapy did not affect the outcome of TB treatment, based on 24 lung cancer patients with co-existing TB (9). Hirashima et al. analyzed 30 metastatic colorectal cancer patients with Mycobacterium tuberculosis (MTB) or Mycobacterium kansasii (MK) infection. They found that it was safe and effective for patients to undergo chemotherapy 
Table 4 Treatment-related AEs reported in Group A and Group B patients

\begin{tabular}{|c|c|c|c|c|c|c|}
\hline Adverse event & \multicolumn{3}{|c|}{ Group A (n=66), n (\%) } & \multicolumn{3}{|c|}{ Group B (n=33), n (\%) } \\
\hline Any adverse event & $30(45.6)$ & $17(25.8)$ & $9(13.6)$ & $15(45.5)$ & $14(42.4)$ & $3(9.1)$ \\
\hline Neutropenia & $18(27.3)$ & 9 (13.6) & $8(12.1)$ & $10(30.3)$ & $6(18.2)$ & $2(6.1)$ \\
\hline Anemia & $30(45.6)$ & $16(24.2)$ & 0 & $16(48.5)$ & $11(33.3)$ & 0 \\
\hline Hypohepatia & $17(25.8)$ & 0 & 0 & 8 (24.2) & $1(3.0)$ & 0 \\
\hline
\end{tabular}

AEs, adverse events.

after some time following MTB or MK treatment (15). They performed another retrospective analysis of 30 patients with various solid cancers and co-existing MTB infection, including eight lung cancer patients, and the results showed that combination therapy was safe and feasible (10). Nevertheless, given the small sample sizes, the differing histories of anti-tumor therapy, and the various cancer types, the conclusions reached in these studies warranted further investigation. In the present study, we enrolled treatment-naïve patients who were diagnosed with lung cancer alone or lung cancer with co-existent active $\mathrm{TB}$ and confirmed the safety and effectiveness of the anti-TB treatment combined with chemotherapy. However, little evidence exists on the optimum initiation time for chemotherapy following anti-TB therapy. It has been reported that sputum bacteria turned negative after 2-4 weeks of anti-TB treatment (16), which appears to be the appropriate time to start chemotherapy. But according to our previous clinical practice, chemotherapy has little impact on immunity for patients with solid tumors, and concurrent chemotherapy and tuberculosis treatment seem to be safe and feasible. So in this study, some patients started chemotherapy and anti-TB treatment simultaneously, while others started chemotherapy after 2 weeks of anti-TB treatment. All of these patients achieved sputum conversion and radiographic remission after 3 months of anti-TB therapy. This result suggests that anti-TB treatment concurrent with chemotherapy may be a feasible strategy, but additional large-scale studies are warranted.

Due to hepatic microsomal drug-metabolizing enzymes, drug-induced hypohepatia is one of the most common AEs of both anti-TB treatment and chemotherapy $(17,18)$. Although no increased incidence of druginduced hypohepatia was observed in patients with concurrent chemotherapy and anti-TB therapy. Drug interactions cannot be ignored. Isoniazid inhibits hepatic enzymes, which can delay metabolism and increase the concentration of drugs (17). Conversely, rifampicin induces hepatic enzymes, which can accelerate drug metabolism and reduce plasma drug concentrations, especially for tyrosine kinase inhibitors such as gefitinib and erlotinib $(19,20)$. In this study, patients with a driver gene mutation were excluded. However, in clinical practice, EGFR-mutant lung cancer patients sometimes exhibit co-existent active TB. The incidence of grade 3 hypohepatia caused by EGFRTKIs has been reported to be approximately $1.7-18 \%$ (21). However, there is little information on the AEs of EGFR inhibitors combined with anti-TB treatment. Rifampin should be excluded from anti-TB treatment in EGFRmutant lung cancer patients considering drug metabolism and potential interactions. An alternative strategy is to use compounds metabolized by bile, such as afatinib (22). Polymorphisms in genes encoding hepatic drugmetabolizing enzymes may be related to hypohepatia caused by some drugs $(23,24)$. In the future, it will be possible to create individualized therapies at the genetic level that avoid drug-induced hypohepatia.

Presently, chemotherapy, targeted therapies, and immunotherapy are the mainstays of treatment for advanced non-small-cell lung cancer. Cell-mediated immunity plays a vital role in the development of TB. It has been reported that MTB promotes tumor metastasis by inhibiting the T-cell immune response through the PD-1/PD-L1 pathway (25). However, whether immune surveillance is involved in the development of TB remains unclear. Fujita et al. reported the appearance of TB in a lung cancer patient during PD-1 inhibitor treatment (26), but none of the patients in our study received PD-1 inhibitor treatment. Therefore, results suggested that the efficacy of immunotherapy combined with anti-TB treatment in lung 
cancer patients with co-existent active TB requires further investigation.

There were limitations to our study. First, although propensity score matching has been used to minimize confounding bias, the potential selective bias emanated from the retrospective nature of research cannot be avoided completely. Second, we did not test for the hepatitis B virus (HBV), a common infection in China. The conclusions in this subgroup may be different. Thirdly, subgroup analysis was not performed in patients with drug-resistant $T B$, diabetes, or other autoimmune diseases whose anti-TB treatment was unusual. Thus, our conclusions may not apply to these patients. Additionally, due to the sample size, our study only focused on advanced lung cancer patients receiving first-line chemotherapy. The impact of multiple lines of chemotherapy and $\mathrm{HBV}$ infection were not analyzed. Finally, since only one patient with co-existent TB and lung cancer changed the anti-TB regimen, our study was unable to evaluate whether the adjustment of anti-TB regimen would affect the results of the study.

In conclusion, this study revealed that concurrent firstline chemotherapy and anti-TB treatment in lung cancer patients with co-existent TB did not increase the incidence of AEs such as hypohepatia and hematologic toxicity, and did not affect the efficacy of either the anti-TB therapy or chemotherapy.

\section{Acknowledgments}

Funding: This work was supported by the national scientific and technological projects for the prevention and control of major infectious diseases (grant numbers 2018ZX10715004002-005).

\section{Footnote}

Reporting Checklist: The authors have completed the STROBE reporting checklist. Available at http://dx.doi. org/10.21037/atm-20-5964

Data Sharing Statement: Available at http://dx.doi. org/10.21037/atm-20-5964

Conflicts of Interest: All authors have completed the ICMJE uniform disclosure form (available at http://dx.doi. org/10.21037/atm-20-5964). The authors have no conflicts of interest to declare.
Ethical Statement: The authors are accountable for all aspects of the work in ensuring that questions related to the accuracy or integrity of any part of the work are appropriately investigated and resolved. All procedures performed in this study involving human participants were in accordance with the Declaration of Helsinki (as revised in 2013). The study was approved by the ethics committee of Guangzhou Chest Hospital (No. 2020-21). The requirement of informed consent was waived because of the retrospective nature of the study.

Open Access Statement: This is an Open Access article distributed in accordance with the Creative Commons Attribution-NonCommercial-NoDerivs 4.0 International License (CC BY-NC-ND 4.0), which permits the noncommercial replication and distribution of the article with the strict proviso that no changes or edits are made and the original work is properly cited (including links to both the formal publication through the relevant DOI and the license). See: https://creativecommons.org/licenses/by-nc-nd/4.0/.

\section{References}

1. Leung CC, Hui L, Lee RS, et al. Tuberculosis is associated with increased lung cancer mortality. Int J Tuberc Lung Dis 2013;17:687-92.

2. Yu YH, Liao CC, Hsu WH, et al. Increased lung cancer risk among patients with pulmonary tuberculosis: a population cohort study. J Thorac Oncol 2011;6:32-7.

3. Liang HY, Li XL, Yu XS, et al. Facts and fiction of the relationship between preexisting tuberculosis and lung cancer risk: a systematic review. Int J Cancer 2009;125:2936-44.

4. Parker CS, Siracuse CG, Litle VR. Identifying lung cancer in patients with active pulmonary tuberculosis. J Thorac Dis 2018;10:S3392-7.

5. Tamura A, Hebisawa A, Tanaka G, et al. Active pulmonary tuberculosis in patients with lung cancer. Kekkaku 1999;74:797-802.

6. Lima GC, Silva EV, Magalhães PO, et al. Efficacy and safety of a four-drug fixed-dose combination regimen versus separate drugs for treatment of pulmonary tuberculosis: a systematic review and meta-analysis. Braz J Microbiol 2017;48:198-207.

7. Pontali E, Centis R, D'Ambrosio L, et al. Recent evidence on delamanid use for rifampicin-resistant tuberculosis. J Thorac Dis 2019;11:S457-60. 
8. Eddy J, Khan T, Schembri F. Medical management of drug-sensitive active thoracic tuberculosis: the workup, radiographic findings and treatment. J Thorac Dis 2018;10:S3378-91.

9. Kim DK, Lee SW, Yoo CG, et al. Clinical characteristics and treatment responses of tuberculosis in patients with malignancy receiving anticancer chemotherapy. Chest 2005;128:2218-22.

10. Hirashima T, Tamura Y, Han Y, et al. Efficacy and safety of concurrent anti-Cancer and anti-tuberculosis chemotherapy in Cancer patients with active Mycobacterium tuberculosis: a retrospective study. BMC Cancer 2018;18:975.

11. Global tuberculosis report 2019. World Health Organization. c2019 [cited 2019 Oct 17]. Available online: https://www.who.int/tb/data/en/

12. Eisenhauer EA, Therasse P, Bogaerts J, et al. New response evaluation criteria in solid tumours: revised RECIST guideline (version 1.1). Eur J Cancer 2009;45:228-47.

13. Varol Y, Varol U, Unlu M, et al. Primary lung cancer coexisting with active pulmonary tuberculosis. Int J Tuberc Lung Dis 2014;18:1121-5.

14. Tamura A, Hebisawa A, Sagara Y, et al. Coexistence of lung cancer and active pulmonary mycobacteriosis. Kekkaku 2005;80:413-9.

15. Hirashima T, Nagai T, Shigeoka H, et al. Comparison of the clinical courses and chemotherapy outcomes in metastatic colorectal cancer patients with and without active Mycobacterium tuberculosis or Mycobacterium kansasii infection: a retrospective study. BMC Cancer 2014;14:770.

16. Ho JC, Leung C. Management of co-existent tuberculosis and lung cancer. Lung Cancer 2018;122:83-7.

17. Ramappa V, Aithal GP. Hepatotoxicity Related to Antituberculosis Drugs: Mechanisms and Management. J Clin

Cite this article as: Ye MF, Su S, Huang ZH, Zou JJ, Su DH, Chen XH, Zeng LF, Liao WX, Huang HY, Zeng YY, Cen WC, Zhang XL, Liao CX, Zhang J, Zhang YB. Efficacy and safety of concurrent anti-tuberculosis treatment and chemotherapy in lung cancer patients with co-existent tuberculosis. Ann Transl Med 2020;8(18):1143. doi: 10.21037/atm-20-5964
Exp Hepatol 2013;3:37-49.

18. Iyer L, Ratain MJ. Pharmacogenetics and cancer chemotherapy. Eur J Cancer 1998;34:1493-9.

19. van Erp NP, Gelderblom H, Guchelaar HJ. Clinical pharmacokinetics of tyrosine kinase inhibitors. Cancer Treat Rev 2009;35:692-706.

20. Burk O, Koch I, Raucy J, et al. The induction of cytochrome P450 3A5 (CYP3A5) in the human liver and intestine is mediated by the xenobiotic sensors pregnane $\mathrm{X}$ receptor $(\mathrm{PXR})$ and constitutively activated receptor (CAR). J Biol Chem 2004;279:38379-85.

21. Takeda M, Okamoto I, Nakagawa K. Pooled safety analysis of EGFR-TKI treatment for EGFR mutation-positive non-small cell lung cancer. Lung Cancer 2015;88:74-9.

22. Wind S, Schnell D, Ebner T, et al. Clinical Pharmacokinetics and Pharmacodynamics of Afatinib. Clin Pharmacokinet 2017;56:235-50.

23. Wang PY, Xie SY, Hao Q, et al. NAT2 polymorphisms and susceptibility to anti-tuberculosis drug-induced liver injury: a meta-analysis. Int J Tuberc Lung Dis 2012;16:589-95.

24. Huang YS, Chern HD, Su WJ, et al. Polymorphism of the $\mathrm{N}$-acetyltransferase 2 gene as a susceptibility risk factor for antituberculosis drug-induced hepatitis. Hepatology 2002;35:883-9.

25. Cao S, Li J, Lu J, et al. Mycobacterium tuberculosis antigens repress Th1 immune response suppression and promotes lung cancer metastasis through PD-1/PDl-1 signaling pathway. Cell Death Dis 2019;10:44.

26. Fujita K, Terashima T, Mio T. Anti-PD1 Antibody Treatment and the Development of Acute Pulmonary Tuberculosis. J Thorac Oncol 2016;11:2238-40.

(English Language Editors: J. Brown and J. Chapnick) 
Supplementary

Table S1 Subgroup analysis of treatment-related AEs in groups A and B

\begin{tabular}{|c|c|c|c|c|c|c|c|c|}
\hline \multirow{2}{*}{ Characteristic } & \multicolumn{4}{|c|}{ Group A (n=66) } & \multicolumn{4}{|c|}{ Group B $(n=33)$} \\
\hline & Neutropenia & Anemia & Thrombocytopenia & Hypohepatia & Neutropenia & Anemia & Thrombocytopenia & Hypohepatia \\
\hline \multicolumn{9}{|l|}{ Age, years } \\
\hline$<60$ & $14(42.4 \%)$ & 27 (81.8\%) & 9 (27.3\%) & $10(30.3 \%)$ & $9(56.3 \%)$ & $12(75.0 \%)$ & $3(18.8 \%)$ & $5(31.3 \%)$ \\
\hline$\geq 60$ & $21(63.6 \%)$ & 19 (57.6\%) & $7(21.2 \%)$ & $7(21.2 \%)$ & $9(52.9 \%)$ & $15(88.2 \%)$ & $3(17.6 \%)$ & $4(23.5 \%)$ \\
\hline \multicolumn{9}{|l|}{ Smoking status } \\
\hline Smoker & $28(56.0 \%)$ & $34(68 \%)$ & $15(30.0 \%)$ & $14(28.0 \%)$ & 15 (55.6\%) & $21(77.8 \%)$ & $5(18.5 \%)$ & 7 (25.9\%) \\
\hline \multicolumn{9}{|l|}{ Stage } \\
\hline III & $8(50.0 \%)$ & $13(81.3 \%)$ & $3(18.8 \%)$ & $1(6.3 \%)$ & 7 (77.8\%) & $9(100 \%)$ & $2(22.2 \%)$ & $2(22.2 \%)$ \\
\hline IV & 27 (54.0\%) & $33(66.0 \%)$ & $13(26.0 \%)$ & $16(32.0 \%)$ & $11(45.8 \%)$ & $18(75.0 \%)$ & $4(16.6 \%)$ & 7 (29.2\%) \\
\hline \multicolumn{9}{|l|}{ Pathology } \\
\hline Adenocarcinoma & $12(37.5 \%)$ & $21(65.6 \%)$ & $5(15.6 \%)$ & $10(31.3 \%)$ & $7(41.2 \%)$ & $13(76.6 \%)$ & $3(17.6 \%)$ & $6(35.3 \%)$ \\
\hline Squamous-cell carcinoma & $13(65.0 \%)$ & $17(85.0 \%)$ & $6(30.0 \%)$ & $4(20.0 \%)$ & $5(50.0 \%)$ & $9(90.0 \%)$ & $2(20.0 \%)$ & $2(20.0 \%)$ \\
\hline SCLC & $10(71.4 \%)$ & $8(57.1 \%)$ & $5(35.7 \%)$ & $3(21.4 \%)$ & $5(83.3 \%)$ & $5(83.3 \%)$ & $1(16.7 \%)$ & $1(16.7 \%)$ \\
\hline
\end{tabular}

AEs, adverse events; SCLC, small cell lung cancer. 